INGENIERIA CIVIL

\title{
Desarrollo de un modelo computacional para la evaluación mecánica y funcional de la articulación trapecio metacarpiana
}

CIVIL ENGINEERING

\section{Development of a computational model for the mechanical and functional evaluation of the trapeziometacarpal joint}

\author{
Fernando Ramirez*, Carlos M. Piscal**§, Andrés F. Guzmán***, Constanza L. \\ Moreno**** \\ * Departamento de Ingeniería Civil y Ambiental, Universidad de los Andes, Bogotá, Colombia. \\ **Programa de Ingeniería Civil, Universidad de la Salle, Bogotá, Colombia. \\ ***Departamento de Ingeniería Civil y Ambiental, Universidad del Norte, Barranquilla, Colombia. \\ ****Hospital Universitario, Fundación Santa Fe de Bogotá, Bogotá, Colombia. \\ §cpiscal@unisalle.edu.co,framirez@uniandes.edu.co,faguzman@uninorte.edu.co,clmorenos@ \\ yahoo.com
}

Recibido: 30 de Octubre de 2012 - Aceptado: 27 de Septiembre de 2013

\section{Resumen}

Se desarrolló un modelo computacional del pulgar con el fin de evaluar los esfuerzos y deformaciones a los que se en $\neg$ cuentra sometida la articulación trapecio metacarpiana. Utilizando tomografías axiales computarizadas se rea lizó la reconstrucción de la geometría 3D del pulgar, y con base en un análisis anatómico y funcional se generó un modelo computacional para simular el comportamiento de la articulación bajo condiciones normales de servi $\neg$ cio. Del análisis del modelo por el método de elementos finitos, se obtuvieron las fuerzas musculares y la distribución de esfuerzos, para condiciones normales de funcionamiento y en posiciones anatómicas específicas de agarre.

Palabras clave: Articulación trapecio metacarpiana (TMC), elementos finitos, modelo computacional, pulgar.

\begin{abstract}
A computational model for the thumb was developed in order to evaluate the stresses and strains in the Trapeziometacarpal joint under physiological conditions. A reconstruction of the $3 \mathrm{D}$ geometry of the thumb was performed through image processing of computerized axial tomographies. Based on anatomical and functional analysis of the thumb, a computational model was generated to simulate the behavior of the joint under normal working conditions. The tendon forces and the distribution of stresses in the joint were obtained by means of mechanics analysis using the finite element method; these analyses were performed for regular usage conditions and specific anatomical grip positions.
\end{abstract}

Keywords: Computational model, finite element method, thumb bone, Trapeziometacarpal joint (TMC). 


\section{Introducción}

La principal función de la mano es la prensión, siendo perfecta en el hombre debido a las características que tiene el pulgar para lograr la oposición con respecto al resto de los dedos, Grimm et al. (2002). El pulgar asume entre el 40 y el $50 \%$ de la fun $\neg$ ción de la mano siendo la articulación trapecio-metacarpiana la más importante de su complejo osteoarticular. La especial configuración anatómica de esta articulación permite la formación de pinzas entre el pulgar y los dedos largos además de la realización de una empuñadura potente, explicando así su gran importancia funcional, Jara \& Flores (2004). La gran cantidad de movimientos y esfuerzos a los que está sometida la hacen susceptible a lesiones frecuentes tanto traumáticas como de-generativas. Estas lesiones son consecuencia del desgaste del cartílago articular que conlleva a la disminución de la movilidad y limitación de la función normal de la mano, que usualmente requiere intervención médico-quirúrgica.

En la actualidad se han desarrollado múltiples procedimientos para tratar de restablecer las funciones normales de esta articulación, sin embargo no se tiene conocimiento específico de la eficacia relativa de ellos ni de su escenario específico de aplicación.

Dada la importancia de la articulación Trapecio Metacarpiana y su alto nivel de afectación, se hace necesario un conocimiento detallado de los esfuerzos que esta experimenta en condiciones normales e intervenidas, para lo cual como una nueva herramienta de la medicina moderna se desarrollan los modelos computacionales, los cuales permiten simular el comportamiento de estructuras anatómicas facilitando la realización de estudios mucho más diversos, flexibles y sobre todo, no invasivos.

En este trabajo se presenta un modelo computacional del pulgar para evaluar los esfuerzos y de-formaciones a los que se encuentra sometida la articulación Trapecio Metacarpiana. El modelo presentado incluye la reconstrucción 3D de la geo $\neg$ metría del pulgar y el análisis mecánico usando el método de elementos finitos a partir del cual se encontraron las fuerzas musculares y la distribución de esfuerzos bajo condiciones normales de funcionamiento y en posiciones anatómicas específicas de agarre.

\section{Metodología}

Para el desarrollo de un modelo computacional representativo, no solo del pulgar sino de cualquier estructura anatómica en general, se deben considerar las siguientes etapas:

1) Estudio anatómico y funcional; 2) Reconstrucción de la geometría; 3) Determinación de condiciones de frontera (cargas, apoyos), propiedades de materiales, y tipos de elementos finitos a utilizar en la simulación; 4) Validación del modelo computacional.

\subsection{Estudio anatómico y funcional del pulgar}

Debido a la extensión que implica un estudio de la anatomía y funcionalidad del pulgar, en este documento se presentan los aspectos más sobresalientes e influyentes para la realización de un modelo computacional del mismo.

Las estructuras anatómicas que intervienen directamente en el movimiento del pulgar son: huesos, articulaciones, ligamentos, músculos y tendones; por lo tanto estas estructuras deben tenerse en cuenta para el desarrollo de un modelo mecánico del mismo.

\subsubsection{Huesos}

Los huesos que conforman la estructura ósea del pulgar son: falange distal, falange proximal, primer metacarpiano y los huesos sesamoideos. Para fines del modelo desarrollado en esta investigación se reconstruyó también el Trapecio, el cual pertenece a los huesos del carpo y se articula en su parte distal con el hueso del primer metacarpiano, formando así la articulación Trapecio Metacarpiana (TMC, del inglés Trapeziometacarpal Joint). La importancia del sistema óseo radica en la diversidad de funciones que desarrolla en el cuerpo humano, funciones de tipo estructural tales como dar soporte y forma 
al cuerpo, además de sostener órganos y tejidos blandos; funciones de tipo locomotor resultado de la interacción de los huesos con los músculos y las articulaciones permitiendo el movimiento. Por otra parte el tejido óseo almacena una serie de minerales, especialmente calcio y fosforo, necesarios para la contracción muscular entre otras funciones, Thibodeau \& Patton (2008).

\subsubsection{Articulaciones y ligamentos}

Las articulaciones que forman parte del pulgar son: Articulación Trapecio Metacarpiana, Metacarpofalángica, e Interfalángica. Estas articulaciones se clasifican estructuralmente como sinoviales, ya que constan de una cavidad sinovial en donde los huesos que forman la articulación se unen por medio de la capsula articular que les rodea. Funcionalmente se denominan diartrosis ya que permiten el libre movimiento pero teniendo en cuenta algunas restricciones dadas por las superficies articulares.

Toda articulación sinovial típica consta de 5 elementos no óseos los cuales son: membrana sinovial, cartílago articular, cápsula fibrosa, líquido sinovial y ligamentos. Las anteriores estructuras permiten que las articulaciones desarrollen las siguientes funciones:

a) Servir como estructuras de unión para dos o más huesos próximos; b) Permitir el movimiento de un segmento óseo con respecto a otro; c) Brindar estabilidad restringiendo movimientos no permitidos, Palastanga et al. (2000)

\subsubsection{Articulación trapecio metacarpiana}

Es la articulación que une la cara distal del trapecio con la base del primer metacarpiano, tiene una configuración anatómica en silla de montar única en el cuerpo humano, que se caracteriza por poseer superficies articulares cóncavo-convexas en contraposición y permitir movimientos articulares de abducción, aducción, flexión, extensión y circunducción.

Es una articulación biaxial, permite movimientos en dos planos diferentes, abducción-aducción que se encuentran en el plano de la palma de la mano y flexión-extensión, en el plano perpendicular al plano de la palma de la mano. El movimiento de circunducción es un movimiento circular que resulta posible gracias a la configuración anatómica que posee esta articulación y se caracteriza porque la flexión, extensión, abducción y aducción se realizan de manera consecutiva, Toshikido et al. (1992), Esta articulación tiene tendencia hacia subluxación dorsal y radial, incrementándose la probabilidad cuando las fuerzas de pinza y agarre se aplican, para lo cual posee un grupo de 5 ligamentos (dorso radial, posterior oblicuo, intermetacarpiano, colateral cubital, volar o anterior oblicuo) diseñados principalmente para dar restricciones específicas a los movimientos del pulgar, manteniendo la estabilidad.

El equivalente mecánico para cada articulación se seleccionó estudiando su configuración geométrica, las características de los ligamentos, las restricciones de los tendones y músculos y la función de la articulación, Fitzgerald et al. (2002).

El equivalente mecánico para la articulación TMC es una unión universal con 2 grados de libertad: momento alrededor del eje $\mathrm{z}(\mathrm{Mz})$ permitiendo la flexión - extensión, y momento alrededor del eje y (My) permitiendo la abducción-aducción. Obviamente, el equivalente mecánico escogido es una aproximación; algunas rotaciones adicionales ocurren dando un tercer grado de libertad para lograr una mejor oposición con el pulpejo de los otros dedos, Xu ( 1999).

\subsubsection{Articulación metacarpofalángica}

La Articulación Metacarpofalángica es la que une el primer metacarpiano con la falange proximal. Está conformada por la superficie biconvexa de la cabeza del primer metacarpiano y la superficie cóncava de la falange proximal por lo cual es una articulación de tipo condilar; en este tipo de articulación el cóndilo ovalado (convexo) de un hueso se acomoda en la cavidad elíptica (cóncava) del otro, $\mathrm{Xu}(1999)$. Esta articulación presenta un doble sistema estabilizador: por un lado un sistema capsulo ligamentoso (pasivo) y por otro un sistema muscular (activo); posee ligamentos 
particularmente resistentes y flexibles como lo son los 2 ligamentos colaterales (cubital y radial) además de su ligamento o placa palmar, estos se encargan de restringir los movimientos laterales excesivos pero solo empiezan a ser efectivos después de que la articulación se encuentra entre 10 y 15 grados de flexión con respecto a su posición normal, Xu(1999) . Esta articulación es de tipo biaxial ya que posee 2 grados de libertad. El primero de ellos permite los movimientos de abducción-aducción y el segundo permite los movimientos de flexión-extensión, los fuertes ligamentos colaterales que posee limitan los movimientos de rotación en su propio eje(Torsión). Probablemente la mejor representación mecánica para esta articulación sea un sistema bola-cavidad que permite movimientos libres en todos los planos, pero debido a las restricciones dadas por el complejo ligamentario, algunos de estos movimiento se restringen, llegando a considerar el equivalente mecánico de esta articulación una unión universal con 2 grados de libertad articular, momento alrededor del eje $\mathrm{z}(\mathrm{Mz})$ permitiendo la flexión - extensión, y momento alrededor del eje y (My) permitiendo la abducción-aducción. Este último movimiento está limitado por la anchura del primer metacarpiano y los ligamentos colaterales que este posee, por lo cual se generan únicamente 15 grados de desplazamiento en torno a un eje anteroposterior a través de la cabeza del metacarpiano. Palastanga et al. (2000).

\subsubsection{Articulación interfalángica}

La Articulación Interfalángica permite la unión entre la falange distal y la falange proximal. Es una articulación de tipo troclear (de funcionamiento similar a una bisagra), tiene forma bicondilar, articula la base de la falange distal con el cóndilo de la falange proximal, esta soportada por un sistema capsulo-ligamentoso compuesto por ligamentos colaterales y una placa palmar; la integridad de estos elementos complementados con la capsula dorsal y los músculos extrínsecos representan la clave de la estabilidad articular, Snell (2004). La articulación Interfalángica permite movimientos de extensión entre 15 a 20 grados desde su posición anatómica y cerca de 80 grados en flexión. Esta articulación se representa mecánicamente por un sistema tipo bisagra con un solo grado de libertad articular, momento alrededor del eje z (Mz) permitiendo la flexión extensión. Angulo \& Alvarez (2011).

\subsubsection{Músculos y tendones}

Los músculos son tejidos u órganos caracterizados por su capacidad de contraerse, por lo general en respuesta a un estímulo nervioso, Brand (2000).

Las funciones principales de los músculos son: producir movimientos, ayudar a la estabilidad articular y generar energía mecánica para la transformación de la energía química, Brand (2000).

El pulgar está compuesto por 2 grupos de músculos: los extrínsecos conformados por el flexor largo del pulgar, extensor corto del pulgar, extensor largo del pulgar y abductor largo del pulgar y los intrínsecos que son el flexor corto del pulgar, abductor corto del pulgar, oponente del pulgar y el aductor o aproximador del pulgar. Estos músculos desarrollan funciones específicas dependiendo de la articulación que se esté analizando.

\subsection{Reconstrucción de la geometría 3d}

Para el estudio de los esfuerzos a los que se encuentra sometida la articulación Trapecio Metacarpiana, se generó con base en el estudio anatómico previo, un modelo computacional del pulgar en el cual se reconstruyeron además del trapecio y el metacarpo, las falanges distal y proximal. A pesar de que la articulación en estudio está ubicada en la base del pulgar entre el trapecio y el metacarpo, se hace necesario reconstruir geométricamente las falanges ya que son puntos de inserción de los músculos que intervienen en el movimiento del pulgar generando esfuerzos en la articulación, además, en el extremo superior de la falange distal es donde se reciben directamente las cargas aplicadas para la mayoría de los modelos prensiles humanos (cilíndrica palmar, término-lateral, centralizado, tridigital, posición subterminal). 
En el modelo computacional los ligamentos fueron modelados simulando su función de permitir y facilitar el movimiento dentro de las direcciones anatómicas naturales. Los músculos se modelaron como apoyos fijos ubicados en sus respectivas inserciones, encargados de dar equilibrio al sistema; el cartílago se simuló mediante la acción del desplazamiento de las superficies óseas en contacto sin que se produzca fricción entre ellas y las cargas aplicadas fueron puntuales en el extremo de la falange distal siguiendo el comportamiento de los modelos prensiles humanos.

Para la reconstrucción de la geometría 3D del pulgar se utilizó un estudio de tomografía computarizado que constaba de 179 cortes separados cada $0.6 \mathrm{~mm}$. Cada corte del estudio en Formato TIFF (Tagged Image File Format) se convirtió en una imagen binaria y mediante operaciones morfológicas se obtuvo el contorno de la geometría externa de los huesos en coordenadas planas utilizando el software ImageJ (Figura 1). En muchos casos, y sobre todo en la zona de las articulaciones donde existe gran cantidad de hueso esponjoso, las imágenes son difusas y los contornos deben ser cerrados y perfeccionados manualmente.

Las coordenadas de los contornos obtenidas se convierten en curvas suaves (splines) obteniéndose así para todas las imágenes secuenciales el llamado modelo de alambre.

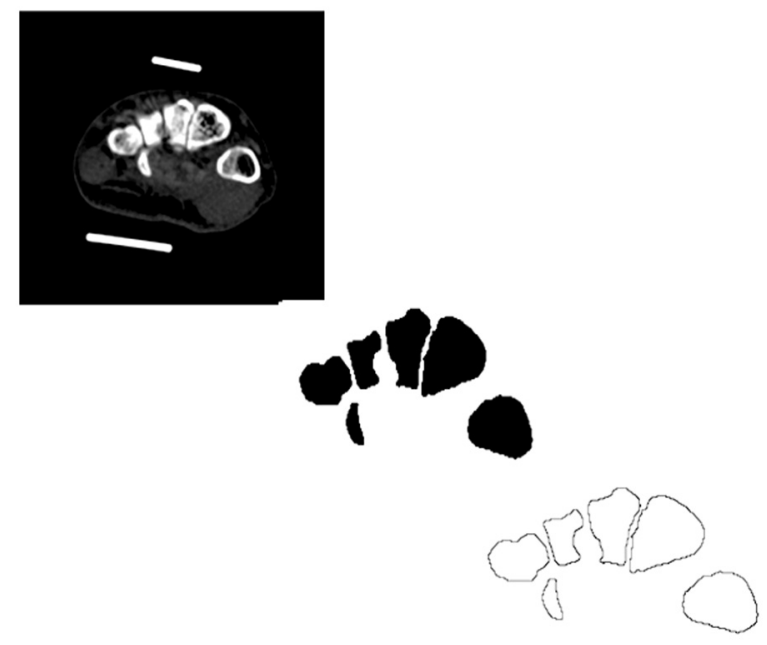

Figura 1. Obtención de contornos

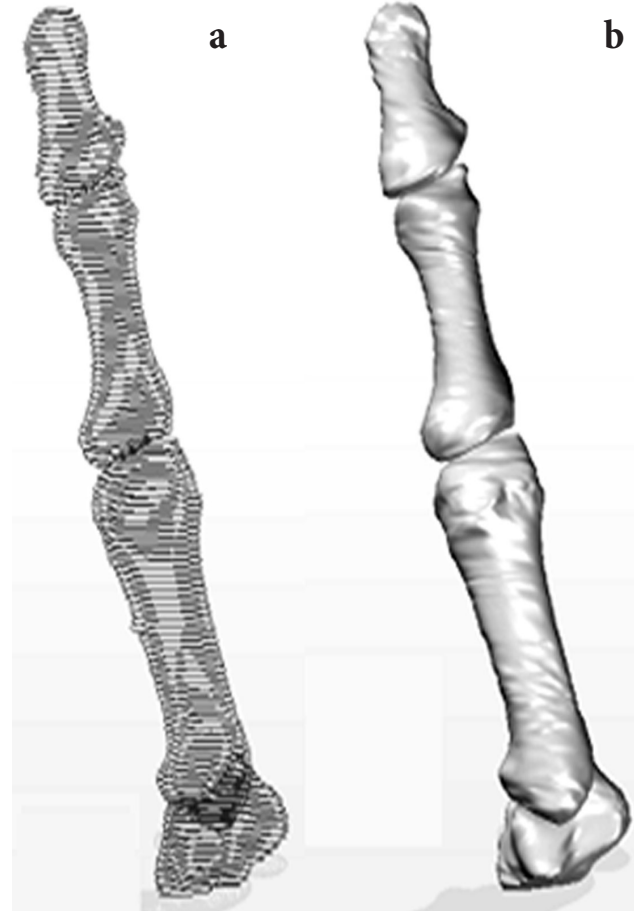

Figura 2. a) Modelo de "alambre" del pulgar, b) Modelo sólido

A partir de los contornos cerrados se crea el volumen mediante operaciones de protrusión disponibles en programas de simulación CAD tal como Solid Edge v.19 (UGS Corp) (Figura 2).

\subsection{Determinación de propiedades de materiales, tipos de elementos, y condiciones de frontera}

\subsubsection{Propiedades de materiales}

Las propiedades mecánicas del hueso no son valores únicos que se puedan predecir de forma precisa, ya que las funciones mecánicas de los diferentes huesos varían, y por lo tanto su micro morfología, Cerrud et al (2005). Estas dependen entonces de factores tales como la distribución mineral, el arreglo estructural, las variaciones entre individuos (edad sexo, padecimientos, grupo antropológico) y la función mecánica del hueso que se está estudiando, Cerrud et al (2005). En esta investigación se buscaba obtener un modelo que se aproxime al comportamiento real de la manera más elemental posible para tener una primera aproximación al entendimiento del problema, por 
lo cual inicialmente se asumió un comportamiento elástico, homogéneo e isotrópico.

Varios autores han hecho trabajos experimentales para encontrar las propiedades mecánicas del hueso humano, entre ellos se puede nombrar: Ashman et. al. (1984), Zysset et. al. (1999), Reilly y Burstein (1975), entre otros, Muller \& Cerrolaza (2001). Teniendo en cuenta los valores encontrados por los anteriores autores, en el modelo computacional desarrollado se trabajó para los huesos un módulo de elasticidad promedio de $17200 \mathrm{MPa}$ y una relación de Poisson de 0.3 .

\subsubsection{Tipos de elementos utilizados en la simulación}

Para el mallado del modelo computacional del pulgar en el Software basado en el método de los elementos finitos Ansys v.10 (ANSYS, Inc), se utilizaron 2 tipos de elementos: Conta174 con 8 nodos y 3 grados de libertad traslacionales por nodo, utilizado para representar el contacto y el deslizamiento al que se encuentran expuestas las zonas de los huesos que hacen parte de las articulaciones interfalángicas, metacarpofalángicas y carpo-metacarpianas; Solid95, tetraédrico, con 20 nodos y 3 grados de libertad traslacionales por nodo empleado para el mallado del resto de las zonas de los huesos.

Los ligamentos se modelaron mediante elementos link10 los cuales tienen la posibilidad de trabajar solo a tensión.

La malla completa generó 30589 elementos sólidos y 44656 nodos (Figura 3).

\subsubsection{Condiciones de frontera}

Con base en el estudio anatómico previo se desarrolló un modelo mecánico del pulgar, en el cual se consideró una condición de empotramiento en la base del trapecio y se modelaron los músculos como apoyos fijos ubicados en sus respectivas zonas de inserción y orientados en sus direcciones correspondientes.

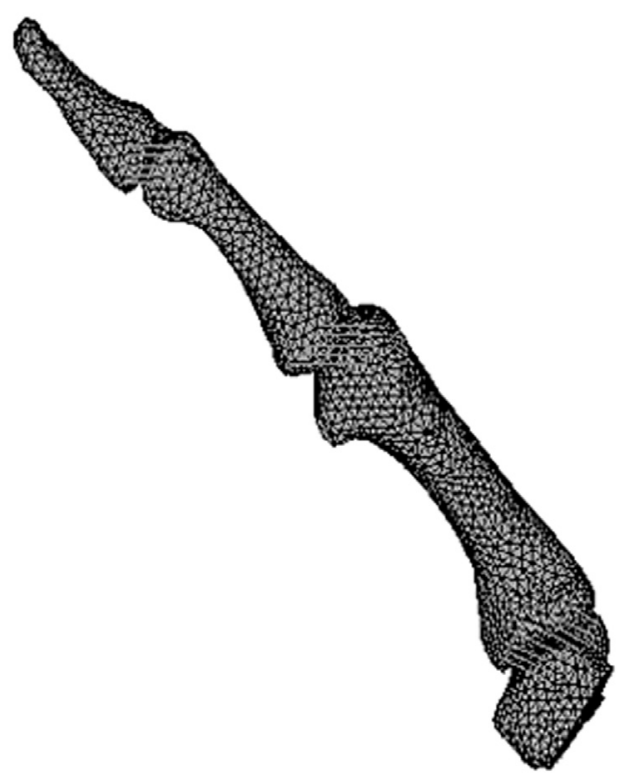

Figura 3. Mallado del modelo del pulgar.

Debido a que el tipo de agarre a simular (términolateral) implica la flexión del pulgar, se modelaron los musculos agonistas y sinergistas de dicho movimiento, los cuales son descritos a continuación: Flexor largo del pulgar (FPL), Aductor del Pulgar (ADD), Abductor corto del pulgar (APB), Flexor corto del pulgar (FPB), Oponente del pulgar (OPP) y Abductor largo del pulgar.

Los ligamentos se modelaron como cables que unen los huesos entre sí y trabajan solo a tensión; se orientaron en la dirección de las fibras que componen los ligamentos.

Teniendo en cuenta las cargas a las que se encuentra expuesta la articulación en condiciones normales de servicio, Xu (1999), se aplicó una carga de 20 $\mathrm{N}$ localizada en el extremo superior de la falange distal (Figura 4).

\section{Resultados y discusión}

Por medio del análisis del modelo se obtuvieron los valores de las reacciones en los apoyos que son equivalentes a las fuerzas musculares necesarias para generar una fuerza de agarre de $20 \mathrm{~N}$ (Tabla 1).

Otro aspecto de gran importancia es la distribución de esfuerzos a los que se encuentra sometida 
Tabla 1. Fuerzas en los Músculos (N) para un agarre tipo término-lateral

\begin{tabular}{ccc}
\hline Músculo & $\begin{array}{c}\text { Modelo } \\
\text { computacional } \\
\text { Reacción (N) }\end{array}$ & $\begin{array}{c}\text { Xu(1999) } \\
\text { Reacción (N) }\end{array}$ \\
\hline$F P L$ & 89.58 & $66,8 \pm 15.8$ \\
$O P P$ & 42.36 & $36.9 \pm 11.7$ \\
$A D D$ & 33.20 & $24.9 \pm 10.2$ \\
$F P B$ & 37.82 & $33.8 \pm 10.9$ \\
$A P B$ & 52.35 & $33.8 \pm 10.9$ \\
$A P L$ & 20.65 & $16.9 \pm 17.9$ \\
\hline
\end{tabular}

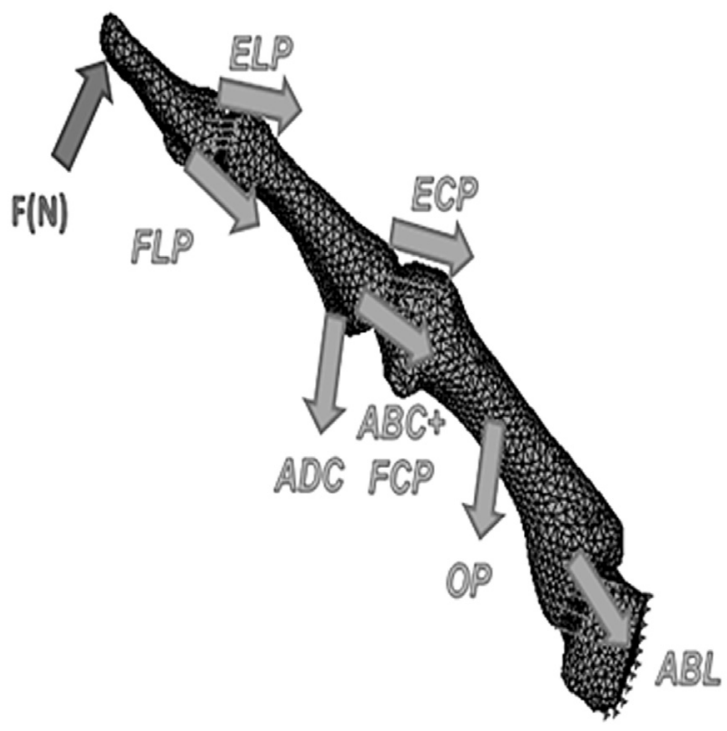

Figura 4. Modelo Mecánico del pulgar

la articulación Trapecio Metacarpiana bajo condiciones normales de servicio y en posiciones anatómicas específicas (Figura 5).

El objetivo de la presente investigación era generar un modelo computacional que simulara adecuadamente el comportamiento de la articulación Trapecio Metacarpiana bajo condiciones normales de funcionamiento. Por lo tanto, se hace necesario validar los resultados obtenidos en este trabajo con resultados de modelos físicos, tales como los presentados por $\mathrm{Xu}$ (1999) en su trabajo, en el cual para funciones anatómicas específicas de prensión se encontraron las combinaciones musculares del pulgar necesarias para poder reproducir una fuerza de agarre de $20 \mathrm{~N}$.

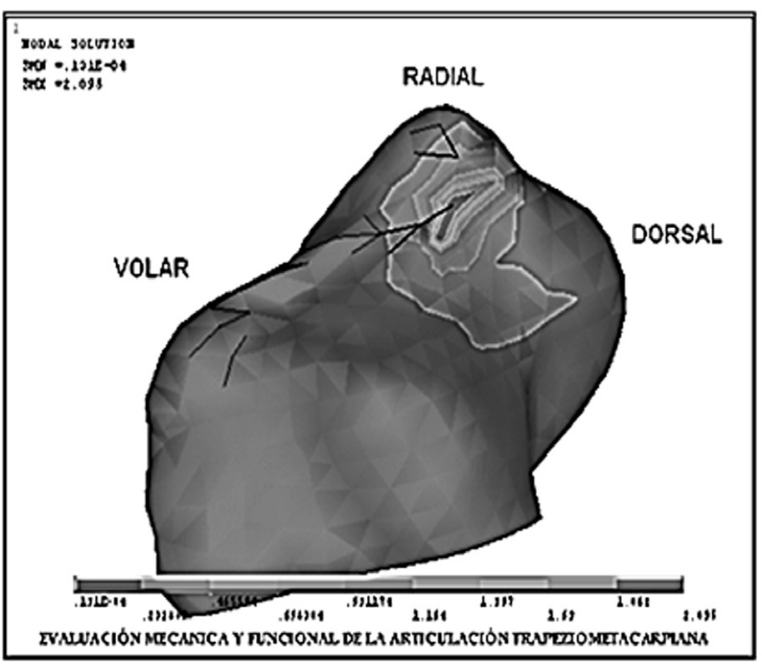

Figura 5. Esquema de distribución de esfuerzos en el Trapecio

La Tabla 1 muestra los resultados obtenidos por Xu (1999) para un agarre tipo término-lateral.

\section{Conclusiones}

Comparando los valores de la fuerzas musculares obtenidos en el modelo computacional con los obtenidos en un modelo físico (Tabla 1), podemos observar que para la condición especifica de agarre término- lateral y con una carga $20 \mathrm{~N}$ en el extremo superior de la Falange distal, se obtuvieron resultados cercanos en los 2 tipos de modelos. Validando así, el trabajo realizado en esta investigación.

La Figura 5 muestra que, para la condición de prensión modelada, los esfuerzos se concentran principalmente en la zona Dorso-Radial del trapecio.

Los avances en el campo de la ingeniería biomédica aumentan las posibilidades de la aplicación de una medicina menos invasiva y más eficiente.

Lareconstrucción tridimensionaldemiembrosóseos a partir de Tomografías Axiales Computarizadas permite generar modelos computacionales para simular y estudiar el comportamiento de los huesos en condiciones normales de servicio. Además, estos modelos permiten dirigir el diseño y evaluación de futuras prótesis. 
Es de suma importancia como siguiente etapa de investigación, realizar modelos computacionales del pulgar en diferentes tipos de agarres anatómicos para un mejor entendimiento del comportamiento de la articulación. Por otra parte sería de gran ayuda para la medicina moderna el poder simular los procedimientos quirúrgicos que buscan restablecer el funcionamiento normal de articulaciones afectadas para evaluar su eficacia y escenario específico de aplicación sin tener que recurrir a procedimientos invasivos.

\section{Referencias bibliográficas}

Abrahams, P., \& Sandy, M. (2005). Gran atlas McMINN de anatomía humana. Barcelona: Océano Mosby.

Angulo, M., \& Álvarez, P. (2011). Biomecánica clínica, Biomecánica de la Extremidad Superior Exploración de la Mano. Reduca, Serie Biomecánica clínica 3 (4), 1-21.

Badia, A., \& Riaño, F. (2005). Osteoartritis de la articulación carpometacarpiana del pulgar. Una clasificación artroscópica original y algoritmo de tratamiento. Revista Colombiana de Ortopedia y Traumatología 19 (2), 54-60.

Brand, P., \& Hollister, A. (1999). Clinical Mechanics of the hand. St.Louis: Mosby.

Cerrud, S., Narvaez, M., Munoz, V., \& Schouweenars, R. (2005). Modelado del comportamiento mecánico del hueso, análisis de los efectos del grado de hidratación. Ingeniería Mecánica Tecnología y Desarrollo 1 (3), 223-232.

Fitzergald, R., \& Herbert, K. Arthur, M. (2004). Ortopedia. España: Panamericana.

Giraldo, C. (2001). Revisión anatómica y biomecánica de miembro superior. Fisioterapia y rehabilitación (3), 1-9.

Grim, M., Arroyo, A., \& Nechyba, M. (2009). A Robotic hand with Realistic Thumb pronation.
Machine intelligence laboratory, University of Florida, Department of Electrical and Computer Engineering.

Jacobsen, S., Wood, J., Knutti, D., \& Biggers, K. (1986). Dextrous Robot Fingers with Desirable Kinematic Forms. International Journal of Robotics Research 17, 996-1012.

Jara,H., \& Flores,A.(2004). Rizartrosis. Reumatologia. 20 (1), 16-23.

Kapit, W., \& Lawrence, E. (2001). Anatomía libro de trabajo. Barcelona: Mc Graw Hill.

Martinez, G. (2006). Artritis Reumatoide. inFARMAte $2(9), 1-9$.

Liangfeng, X. (1999). Biomechanics of the thumb carpometacarpal joint:Topography, ligamentous structure, articular contact, and kinematics. Doctoral Thesis, Columbia University.United States.

Muller, C., \& Cerrolaza, M. (2001). Un nuevo método para la simulación de la estructura ósea mediante la versión $\mathrm{p}$ de elementos finitos. IMME 39(3), 1-32.

Palastanga, N., Field, D., \& Soames, R. (2000). Anatomia y movimiento humano, estructura $y$ funcionamiento. España: Editorial Paidotribo.

Paulsen, F., Putz, R., Reinhard V., \& Sobotta, J. (2004). Atlas de Anatomía humana. España: Panamericana.

Rouviere, H., Delmas, A., \& Delmas, V. (2006). Anatomía Humana. España: Editorial Masson.

Sociedad española de reumatología. (2006). Artritis Reumatoide: Guía de la enfermedad para el paciente. Madrid: Jarpyo Editores.

Snell, R. (2004). Anatomía clínica para estudiantes de Medicina. México: Mc Graw Hill.

Tevar, P.(2001). La cartilla del artrosico: información artrosis de Mano. Madrid: Aula Médica. 
Thibodeau, G., \& Patton, K. (2008). Estructura y función del cuerpo humano. España: Elsevier Inc.

Toshikido, I., Kai-Nan, A., \& Cooney, W. (1992). Functional anatomy and biomechanics of the thumb. Hand clinics 8(1), 9-15.
Valero, F., Johanson, E., \& Towles, J. (2003). Towards a realistic biomechanical model of the thumb: the choice of kinematic description may be more critical than the solution method or the variability/uncertainty of musculoskeletal parameters. Journal of Biomechanic 36, 1019-1030. 